\title{
Thermogenic profiling using magnetic resonance imaging of dermal and other adipose tissues
}

\author{
Ildiko Kasza, ${ }^{1}$ Diego Hernando, ${ }^{2}$ Alejandro Roldán-Alzate, ${ }^{2,3}$ Caroline M. Alexander, ${ }^{1}$ \\ and Scott B. Reeder ${ }^{2,4,5,6,7}$ \\ ${ }^{1}$ McArdle Laboratory for Cancer Research, 'Department of Radiology, ${ }^{3}$ Department of Mechanical Engineering, \\ ${ }^{4}$ Department of Medical Physics, ${ }^{5}$ Department of Biomedical Engineering, ${ }^{6}$ Department of Medicine, and \\ ${ }^{7}$ Department of Emergency Medicine, University of Wisconsin-Madison, Madison, Wisconsin, USA.
}

\begin{abstract}
Dermal white adipose tissue (dWAT) was recently recognized for its potential to modify whole body metabolism. Here, we show that dWAT can be quantified using a high-resolution, fatspecific magnetic resonance imaging (MRI) technique. Noninvasive MRI has been used to describe adipocyte depots for many years; the MRI technique we describe uses an advanced fat-specific method to measure the thickness of dWAT, together with the total volume of WAT and the relative activation/fat depletion of brown adipose tissues (BAT). Since skin-embedded adipocytes may provide natural insulation, they provide an important counterpoint to the activation of thermogenic brown and beige adipose tissues, whereby these distinct depots are functionally interrelated and require simultaneous assay. This method was validated using characterized mouse cohorts of a lipodystrophic, dWAT-deficient strain (syndecan-1 KO) and 2 obese models (diet-induced obese mice and genetically obese animals, ob/ob). Using a preliminary cohort of normal human subjects, we found the thickness of skin-associated fat varied 8-fold, from $0.13-1.10 \mathrm{~cm}$; on average, this depot is calculated to weigh $8.8 \mathrm{~kg}$.
\end{abstract}

Conflict of interest: The University of Wisconsin receives research support from GE Healthcare.

Submitted: February 29, 2016 Accepted: July 19, 2016 Published: August 18, 2016

\section{Reference information:} JCI Insight. 2016;1(13):e87146. doi:10.1172/ji.insight.87146.

\section{Introduction}

Recently, a distinct adipocyte depot has been described that lies within the skin, subjacent to the dermis (1). Although this lipid layer has been frequently grouped together with s.c. white adipose tissue (sWAT), it is now recognized as a distinct depot that responds to different stimuli. Thus, where sWAT, like other WAT depots, is a calorie depot, dermal WAT (dWAT) is known to accumulate in response to ambient cold, hair growth, and bacterial challenge (1-3).

At least for mice, this layer adjusts to different environmental temperatures, such that it is thinner in warm, thermoneutral housing temperatures (regularly $30^{\circ} \mathrm{C}-34^{\circ} \mathrm{C}$ ) and thicker at typical housing temperature $\left(20^{\circ} \mathrm{C}-24^{\circ} \mathrm{C}\right)$. Indeed, lipid is a remarkable biopolymer that is well known to show extremely low heat conductance compared with other polymers with more hydrophilic properties. When dWAT is deficient, mice are underinsulated, and thermogenesis is induced relative to WT mice (4). We calculate that, for a temperature difference of $16^{\circ} \mathrm{C}$ (the difference between mammalian body temperature and a typical housing temperature), the fat present in mouse skin, though only $200-\mu \mathrm{M}$ thick, prevents heat loss by 2 -fold. Thus, the thickness of dWAT is likely to affect how many calories are diverted toward thermogenesis. Metabolic economies have been under intense investigation for a decade, given their potential to combat the obesity epidemic (5-9).

The purpose of this study was to develop and validate an MRI-based noninvasive means of quantitation for dWAT that can be applied to both mice and humans. This technique can be utilized during longitudinal studies so that dynamic changes of all fat depots, including dWAT, can be assessed. We applied chemical shift-encoded (CSE) fat-water separated MRI at 3 Tesla to generate high-resolution cross-sectional images that can be quantified to generate a complete profile of the adipocyte depots that determine net thermogenesis in any given subject. These cross-sectional, fat-only images can be used to measure dWAT thickness; they can also be used to visualize the relative BAT activation and assess the relative lipid content of WAT, along with total WAT volume. In this study, we compared MRI-based fat depot measurements 
to the data derived from the laborious histologic assay of skin sections and other fat depots, which are the usual endpoints for rodent studies. Additionally, we performed a pilot study to assess the thickness of the skin-associated fat depots in the lower leg of healthy human volunteers. A noninvasive and easily available method for profiling of this tissue layer, in parallel with other tissue layers implicated in the thermogenic response, will be particularly important to future studies of human subjects.

\section{Results}

Using an 8-channel wrist coil array and a 3 Tesla clinical MRI scanner (Supplemental Methods are available online with this article; doi:10.1172/jci.insight.87146DS1), BALB/cJ control and lipodystrophic $S d c 1^{-1-}$ $\mathrm{BALB} / \mathrm{cJ}$ mice were imaged using 70 axial imaging slices (Figure 1A; cranial to caudal). Each slice was 0.6-0.7 $\mathrm{mm}$ thick (spaced by $0.1 \mathrm{~mm}$ ); images were generated using a CSE fat-water separation (10). From these images, we could quantify not only the total volume and relative fat content of WAT and BAT depots, but also the thickness of the dWAT embedded in mouse skin, which appears as a clearly defined, separate layer (Figure 1B).

Reconstruction of this layer illustrates the insulating sheath of fat associated with the dWAT layer all around the mouse body (Figure 1C). The patch-wise thick and thin pattern of dWAT accumulation was confirmed by histological analysis (thickest areas reflect patches of skin with active hair growth, or anagen; Figure 1D) (2). To quantify dWAT thickness from these images, rectangular regions of interest (ROIs) were applied along a length of dWAT that was clearly separated from other adipocyte depots (Figure 2A). ROIs larger than the thickness of the fat layer were used to capture the entire lipid content (Figure 2A). The thickness of dWAT was calculated as the fraction of each voxel filled with lipid, assuming the relative signal (and implied lipid density) is approximately the same as a proximal WAT depot (equation is detailed in Methods section). Histological analyses of representative skins from these mouse cohorts are shown in Figure $2 \mathrm{~B}$. We determined that the relative thickness of dWAT measured by both histological assay and MRI was the same (Figure 2C) and that the $S d c 1^{-/-}$skins showed approximately $50 \%$ depletion of dWAT by both methods. Note that data derived by MRI can be separated into measurements of dorsal and ventral skin, where the dWAT on the back skin is typically thicker than on the belly (Figure 2D). Although histological examination is a laborious end-point assay, this technique makes it possible to separate skin domains into nonanagen and anagen stage; dWAT is thickest during hair follicle growth, involuting to a level set by ambient temperature during nonanagen stages of folliculogenesis (Figure 2E).

Furthermore, this technique is able to define the relative lipid density and total volume of individual depots of WAT, using a combination of orthogonal image orientations to identify the limits of each depot, together with the quantitation of WAT by volumetric analysis (a representative image is shown in Figure 3A). Previously, each depot would require laborious dissection from rodent subjects for assay; an example is shown from the work of Cinti and colleagues (Figure 3B) (11). Using MRI, the lipid density and volume for each depot can be assessed simultaneously by image analysis, as follows.

The relative lipid density in BAT and WAT depots was quantified in WT and $S d c 1^{-/-}$mice (Figures 3, C and D). Sdc1 ${ }^{-/-}$mice are lipodystrophic; they show depleted skin-associated lipid (dWAT) and are underinsulated. This results in the chronic activation of thermogenic BAT, with associated depletion of BAT-associated lipid reservoirs. We therefore assessed interscapular BAT (iBAT) depots in $\mathrm{Sdcl}^{{ }^{-1}}$ mice and showed that the signal intensity in BAT was decreased by $40 \%$; this degree of lipid depletion corresponded to that observed by histological staining (Figure 3C). WAT depots show variable recruitment during thermogenic activation (beiging) $(7,8,12,13)$; assessment of the perigonadal WAT depot in $S d c 1^{-/-}$females showed no depletion (Figure 3D), which also matched histological and biochemical assay results.

To investigate whether dWAT thickness is related to the size and lipid content of other WAT depots, we studied dWAT in a rodent model of obesity, the leptin-deficient $o b / o b$ strain (Figure 4) (14). Even for young females (7 weeks old), there is a striking expansion of all WAT depots (Figure 4B) and a 5-fold increase of dWAT, corroborated by both MRI and histological analysis (Figure 4C). The BAT depots in these mice are known to accumulate fat (15), which was confirmed from MRI analysis (Figure 4D).

Since imaging is noninvasive, it can be applied for longitudinal studies of subjects undergoing treatments or dietary modifications. To illustrate this application, C57BL6 males were fed a high-fat diet for 5 weeks to study their individual accumulation of fat in specific adipocyte depots. s.c. adipocyte depots have different properties compared with visceral adipocyte depots; in fact the s.c. depots (exemplified by gluteofemoral fat for humans) are proposed to have positive health benefits $(16,17)$. Therefore, these 2 categories of WAT benefit from independent assay. 

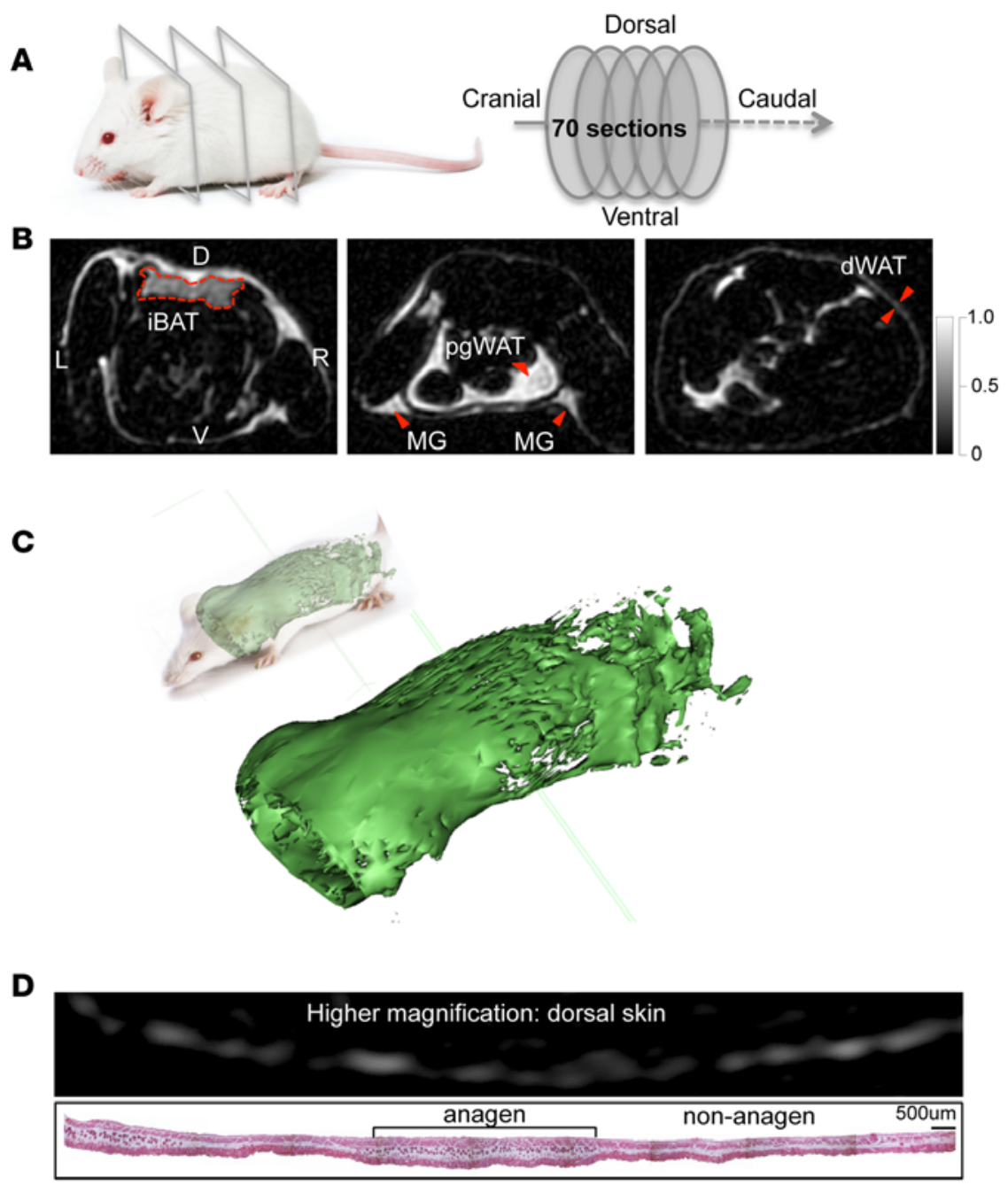

Figure 1. MRI imaging of the sheath of insulating dWAT in mice. (A) Female BALB/c) mice were scanned using a standard 8-channel wrist coil array. Serial transverse images, $700-\mu \mathrm{M}$ thick, were collected for image analysis using clinical OsiriX software. (B) Representative transverse sectional images of mouse bodies; D, dorsal/back; V, ventral/belly; R, right side; L, left side. Depots of interscapular brown adipose tissue (iBAT) were clearly distinguished from proximal white adipose tissue (WAT), including perigonadal WAT (pgWAT) and mammary gland (MC). The skin-associated lipid layer, dermal white adipose tissue (dWAT), was clearly separated from internal adipose depots. The actual size of the transverse sections shown here and in subsequent figures is approximately $3.5 \mathrm{~cm}$. The contrast scale shown at the right was manually set (arbitrary scale $0-3,000$ ). (C) A 3D reconstruction of the body-wide dWAT layer, performed using OsiriX software. (D) Thickness of dWAT varies with various factors; for mice, one of these factors is hair growth. Thus, patches of skin cycle through folliculogenesis (approximately 1 week out of 3; called anagen) (3) and show expanded dWAT (at least 2-fold; see also Figure 2E). Scale bar: $500 \mu \mathrm{m}$.

The C57BL6 male is commonly used for obesity studies because it shows such extreme obese phenotypes, whether induced genetically or by modulation of dietary composition. However, this model, along with others, shows great heterogeneity in the development of white adipose depots. The development of obesity is foreshadowed by gene expression changes recently ascribed to a bistable epigenetic switch (1821). Therefore, it becomes particularly important to perform individualized assessments of adipocyte depot morphogenesis.

Thus, we imaged control and high fat-fed mice and segregated the fat depots into dermal/s.c. fat, as well as visceral and brown adipose (Figures 5 and 6). Measurement of fat tissue volumes showed that dermal/s.c. fat volume was approximately equal to visceral fat volume, and that fat was deposited slowly, and in parallel, for the first 3 weeks of feeding; thereafter, fat deposition accelerates (Figure 5, A-D). When we focused specifically on the kinetics of dWAT accumulation (Figure 6), we found that the accrual of dWAT paralleled the increase in body weight in response to high-fat feeding by both histological and imaging criteria (Figure $6, \mathrm{~A}-\mathrm{C})$. The relative deposition of fat into ventral and dorsal dWAT is shown in Supplemental Figure 5. 
A
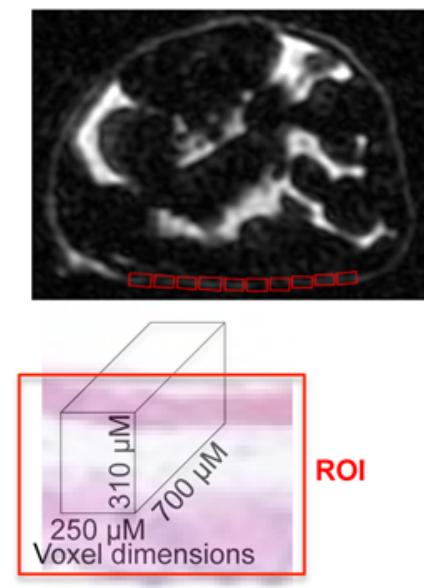

B

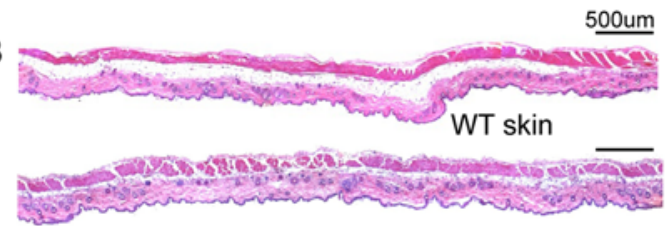

Sdc1-/-
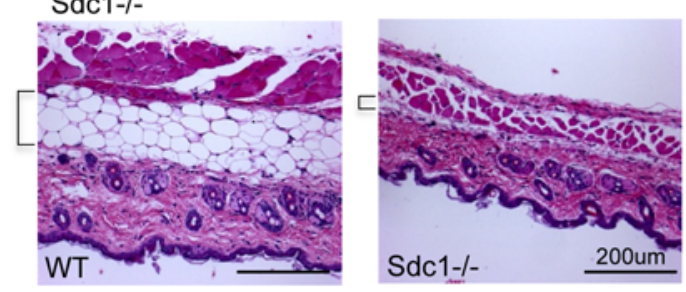

C

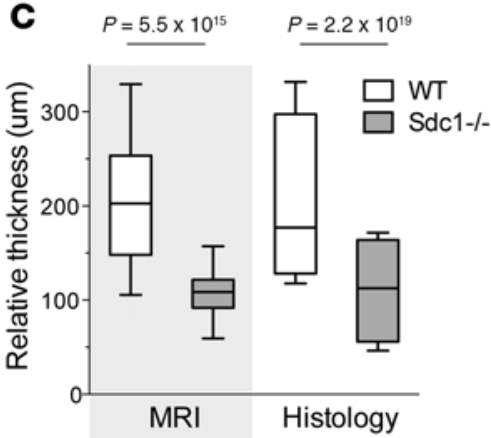

D

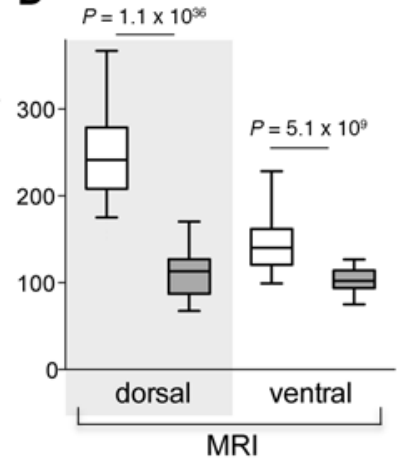

$\mathbf{E}$

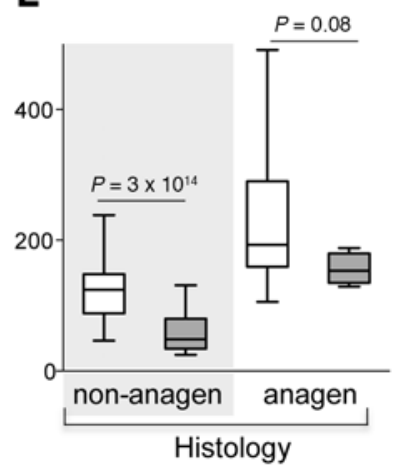

Figure 2. Validation of dWAT imaging. (A) The sampling procedure is illustrated, together with the voxel dimensions used for this study. Typical mouse skin fat layers are less than 1 voxel thick; thus, thickness is calculated based on signal intensity (referenced to a nearby white adipose tissue [WAT] depot) and related to thickness by a volume/ thickness calculation (as described in Methods). ROI, region of interest. (B) The histological appearance of skin samples

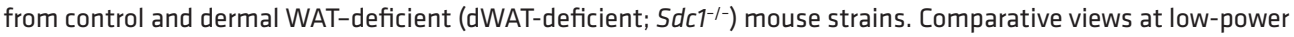
(scale bar: $500 \mu \mathrm{m}$ ) and high-power (scale bar: $200 \mu \mathrm{m}$ ). (C) Relative dWAT thickness, calculated from MRI data, was

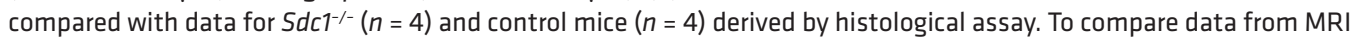
and histological analysis, ventral, dorsal, anagen, and nonanagen skins were pooled. (D) Thickness of dWAT varies with different factors, one of which is body site; here, dorsal (back skin) or ventral (belly skin) are assayed by MRI. (E) A benefit of histological analysis is that dWAT can be measured separately for skin patches in the growth phase of folliculogenesis (anagen, accompanied by the ingression of hair follicles through the dermis and into the dWAT). For the box-and-whisker plots shown in C-E, the box indicates $25 \%-75 \%$, the line is placed at the median, and the whiskers indicate $5 \%-95 \%$. There were no significant outliers (determined by Grubbs' test). Statistical analysis was performed with unpaired 1-tailed $t$ tests using GraphPad Prism software.

The inherent variability in the development of obesity in the C57BL6 cohort revealed these parallels even more strikingly. Thus, although the kinetics of fat accumulation varied from mouse to mouse, it was similar for dWAT and WAT for any given individual; the magnitude of the weight gain was mirrored by increasing dWAT thickness (Figure 6D).

Since the factors affecting metabolism are variable from individual to individual, even for these homozygous groups of mice, this technique becomes extremely useful; it serves to generate more specific data for longitudinal studies. It is, thus, ideal for the evaluation of the heterogeneous metabolic factors present for human subjects. Therefore, we applied this technique to a preliminary cohort of 10 healthy human subjects. Imaging was performed using the same clinical instrument and an 8-channel extremity coil wrapped around the calf region. This region was chosen to reduce the chance of including variable proximal s.c. adipose tissues (see Discussion). Interestingly, the layer of skin-associated fat was highly variable for these 10 individuals, and there was no obvious relationship with BMI, waist/hip ratio, or sex (Figure 7 and Supplemental Figure 2-5). A preliminary questionnaire showed that subjects who self-reported as preferring 
A

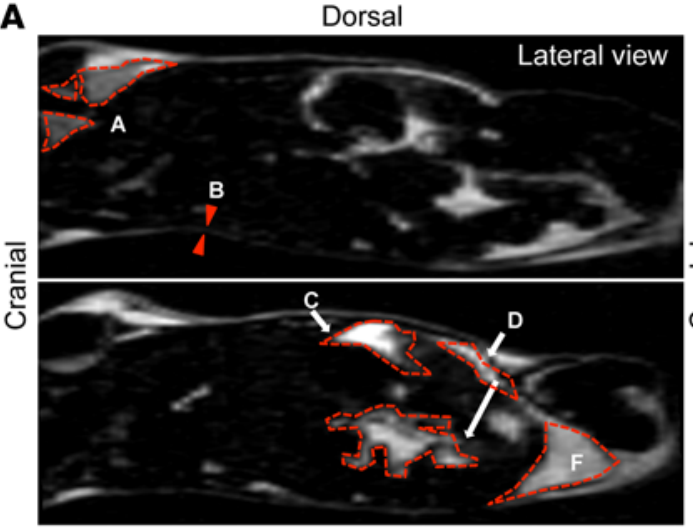

Ventral

C

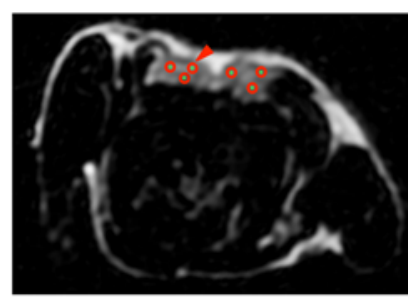

D

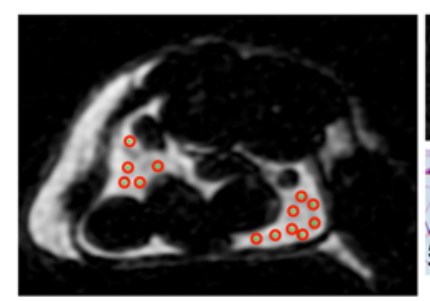

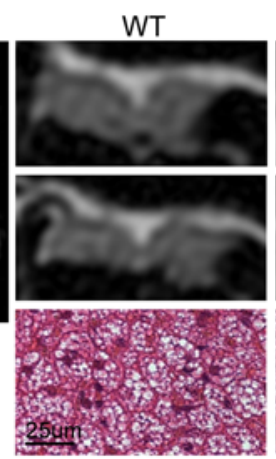

WT

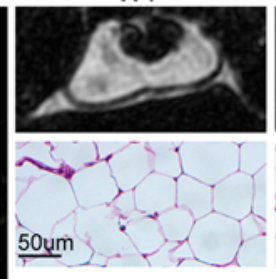

B

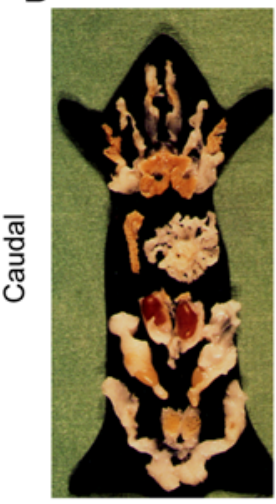

Sdc1-/-

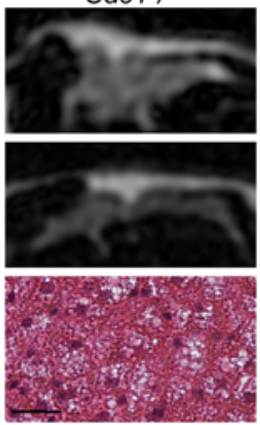

Sdc1-/-

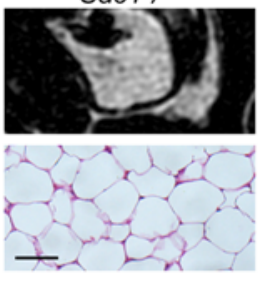

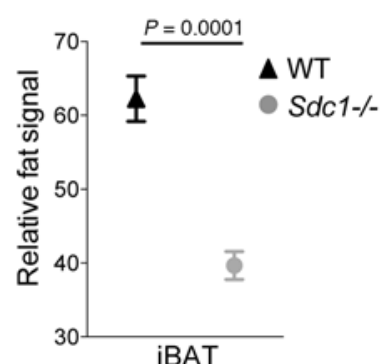

iBAT

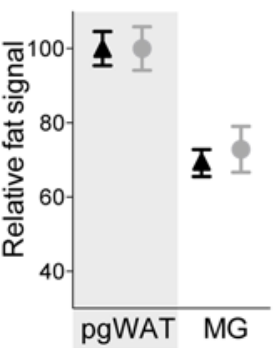

Figure 3. Evaluation of WAT and BAT depots. (A) Lateral views of white adipose tissue (WAT) depots are used to delimit the front and rear of each specific depot, to calculate total WAT volumes. Outlines and arrowheads indicate adipose organs (11): A, anterior subcutaneous; B, dermal; C-E, visceral depots (specifically C, retroperitoneal; D, mesenteric; E, abdomino-pelvic); and F, posterior s.c. The lateral views shown here are approximately $5.5 \mathrm{~cm}$ actual width. (B) This noninvasive method can be compared with previous studies of WAT volumes and appearance, performed using dissection procedures (11). (C) The relative fat signal from interscapular brown adipose tissue (iBAT) depots (normalized to fat signal from perigonadal WAT) was quantified ( $20 \mathrm{ROIs}$ each, $n=3$ ) and validated by comparison with the iBAT depots of coldstressed Sdct-1- mouse strain, previously shown to have depleted lipid content. The relative signal is reported in arbitrary units. The histological appearance of the iBAT depots illustrates the relative lipid depletion (clear droplets on H\&E-stained images); scale bar: $25 \mu \mathrm{m}$. (D) Similarly, the relative fat signals from WAT depots (pgWAT and mammary gland [MG]) were compared for control and $S d \mathrm{ct}^{-1-}$ mice (20 voxels selected at random, indicated for this example in red circles; $n=3$ ). They showed no difference, which confirms the histological data; scale bar: $50 \mu \mathrm{m}$. Dot plots (C and $\mathbf{D}$ ) show mean values; error bars indicate \pm SEM. Statistical analysis was performed with unpaired 1-tailed $t$ tests using GraphPad Prism software.

warmer temperatures than colleagues and family members (perhaps paradoxically) showed a significantly thicker layer of skin-associated fat.

\section{Discussion}

This study demonstrates that chemical shift-encoded, MRI-based fat-only imaging enables assessment of multiple fat depots and that the data derived from MRI matches the results obtained by exhaustive histologic analysis of skins, BAT, and WAT. This technique will allow biomedical researchers to assess dWAT for any given group of rodent models and human subjects or patients, and it will allow them to evaluate 
A

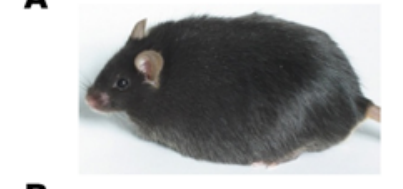

B
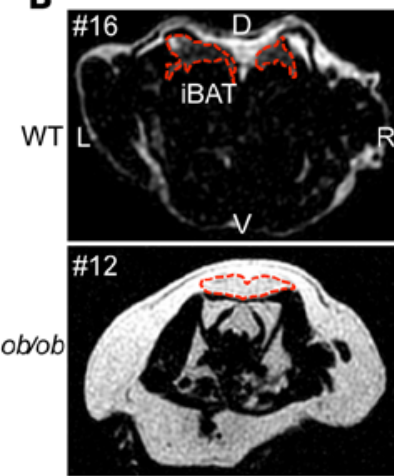

C

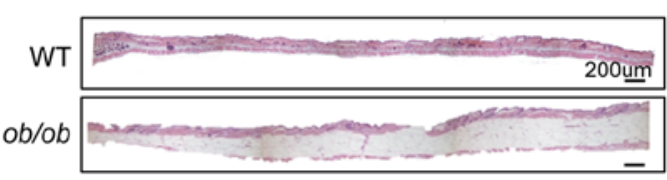

\section{D}

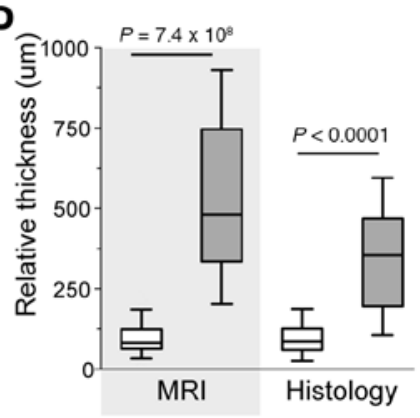

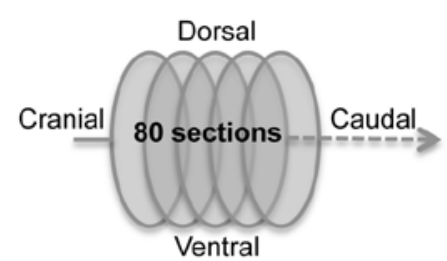
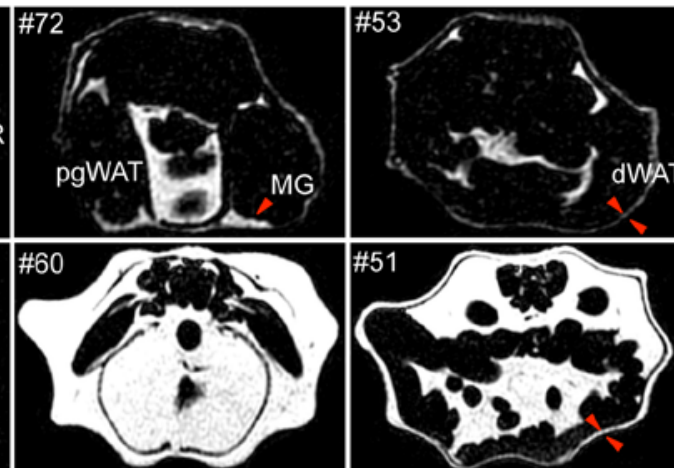

$\square[1.0$

$-0.5$

0
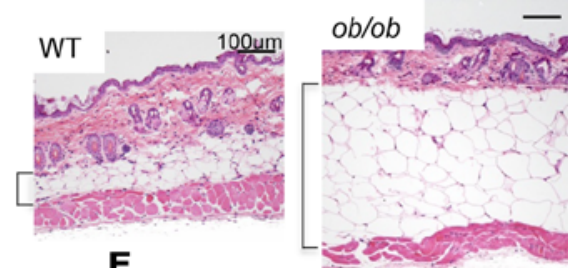

E
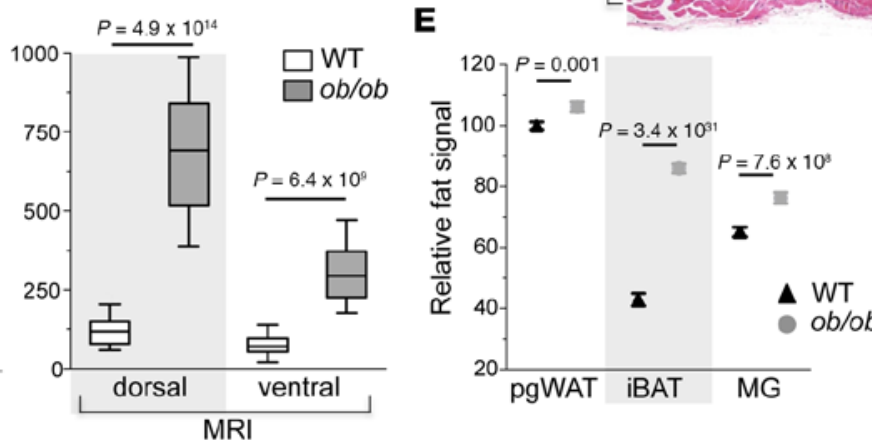

Figure 4. Assay of obese mice. (A) An expanded sampling scheme was applied to 7-week-old female leptin-deficient $o b / o b$ mice (used extensively as a model of obesity). (B) MRI imaging of WT and ob/ob obese mice is shown to illustrate the accumulation of white adipose tissue (WAT) present in these mice. However, the dermal WAT (dWAT) layer is clearly distinguished in central abdominal image sections (shown with red arrow heads). iBAT is outlined in a dotted red line. (C) Comparative histology of skins of control and obese mice, illustrated at low-power magnification, left side (scale bar: $200 \mu \mathrm{m}$ ), and higher power, right side (scale bar: $100 \mu \mathrm{m}$ ). (D) Comparison of relative dWAT thickness in ob/ob mice $(n=4)$ by MRI and histological assays (left panel, data pooled from assay of ventral, dorsal, anagen, and nonanagen skins). The difference between dorsal and ventral dWAT thickness is shown on right side. Box-and-whisker plots are presented as described in Figure 2E. (E) The average density of fat for WAT (periovarian, intra-abdominal) and mammary gland (MG; s.C., specialized) was measured, together with interscapular brown adipose tissue (iBAT). Dot plots are presented as for Figure 3. Statistical analysis was performed with unpaired 1-tailed $t$ tests using GraphPad Prism software.

whether dWAT thickness is affected in patients with skin pathologies or whether it correlates with other physiologies, such as the development of inflammatory obesity or susceptibility to BAT activation. We have validated this technique for rodent models that have thick and thin dWAT and have quantified dWAT in 2 mouse models of obesity (one induced by high-fat diet, the other genetically). In both obese models, the accumulation of fat in dWAT parallels the accumulation of visceral fat. Over the course of 5 weeks of high-fat feeding, we have shown that the visceral WAT, s.c. WAT, and dWAT tend to grow in parallel, with a sharp uptick after 3 weeks. This noninvasive longitudinal imaging method also reveals how fat depots react during the heterogeneous development of obesity in genetically homozygous mouse populations. This offers the opportunity to identify important time points to evaluate molecular factors that determine 
A

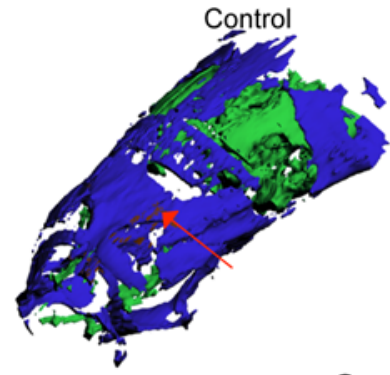

B

C
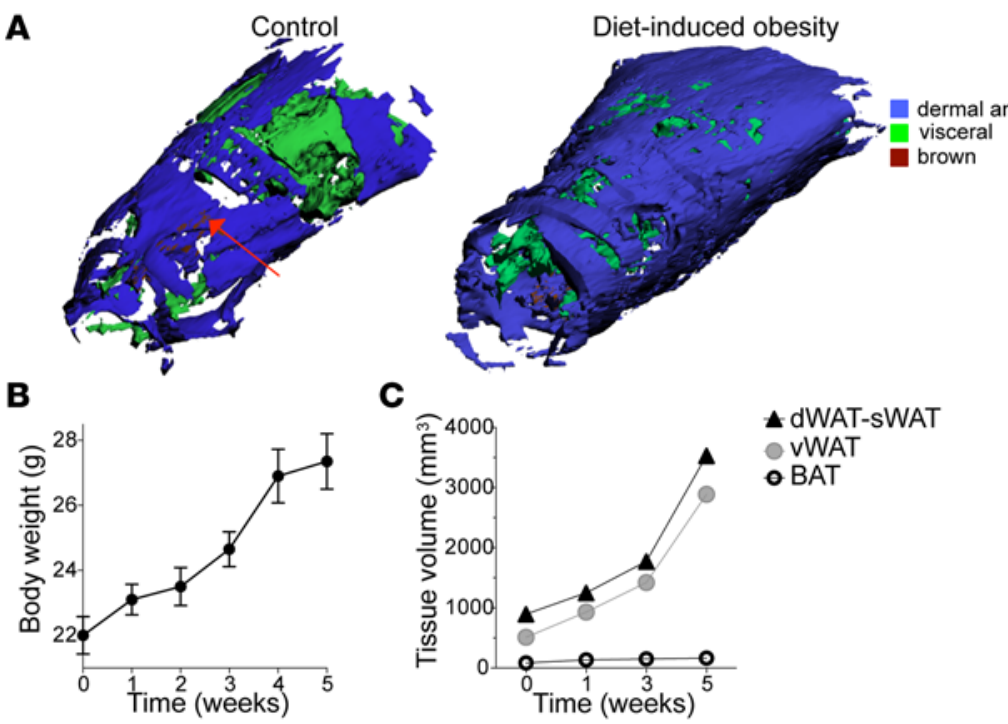

है 4000

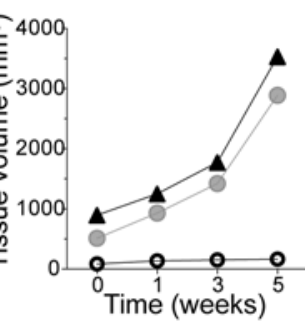

$\triangle$ dWAT-SWAT

VWAT

- BAT

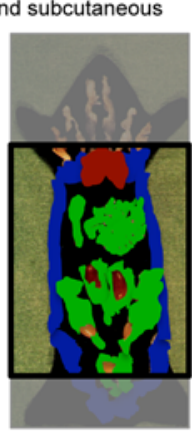

D

w0

w1

w3

w5

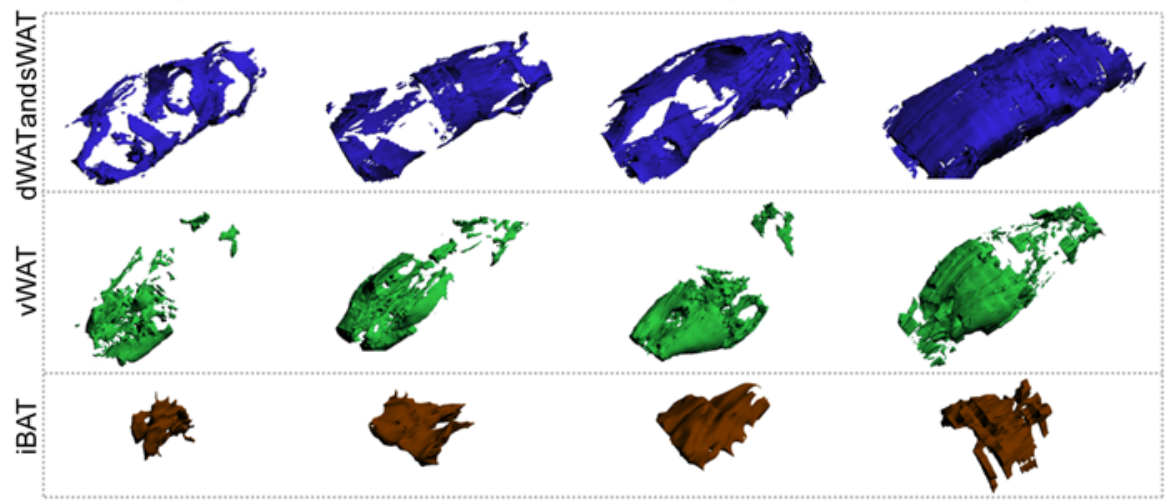

Figure 5. Accumulation of fat in specific adipose depots during high-fat feeding. (A) 3D reconstruction of adipose tissues in mice fed a high-fat diet for 5 weeks, compared with control mice on chow diet, segregated as indicated in the key at the right side (dermal/s.c., dWAT/sWAT; visceral, vWAT; and brown adipose tissue, iBAT; notice that kidneys are also colored brown in key). (B) Body weights of C57BL6 male mice were measured during a 5-week period of high-fat feeding ( $n=22)$. (C and $\mathbf{D})$ During this 5-week administration period, the specific volume of each type of adipose depot was assayed and illustrated visually for 1 mouse, which was imaged sequentially (week 0 to week 5; w0, w1, w3, w5) Statistical analysis was performed with unpaired 1-tailed $t$ tests using GraphPad Prism software.

the promotion of obesogenesis and to stratify populations as obesity resistant or susceptible to evaluate the regulation of adiposity.

Obviously, longitudinal studies of individual human subjects are best served by imaging modalities. We anticipate that this technique could be useful to assess the response of dWAT (and other components of the thermogenic circuit) to interventions, such as changes in diet, drug treatments, and/or environmental conditions. Further, the lack of ionizing radiation and widespread availability of clinical MRI scanners makes these methods well suited for human studies, especially given high inherent genetic and environmental variability.

Intriguingly, evaluation of a preliminary cohort of human subjects suggests that this layer is highly variable, varying from $0.13 \mathrm{~cm}$ to $1.10 \mathrm{~cm}$. If we assume that this layer exists over the entire body, these depots are estimated to weigh approximately $2.1-17.6 \mathrm{~kg}$ (based on an average surface area of $1.8 \mathrm{~m}^{2}$ ). Note that the Reference Man $(70 \mathrm{~kg})$ is estimated to have $15 \mathrm{~kg}$ of adipose tissue sequestered into visceral depots (22). There are remarkable implications of such variability in the thickness of dWAT; an 8-fold range of skin-associated fat would be expected to show an 8-fold difference in heat loss and relative insulation, with proportional metabolic economies. Thus, the proportion of time spent by a human subject at nonthermo- 
A
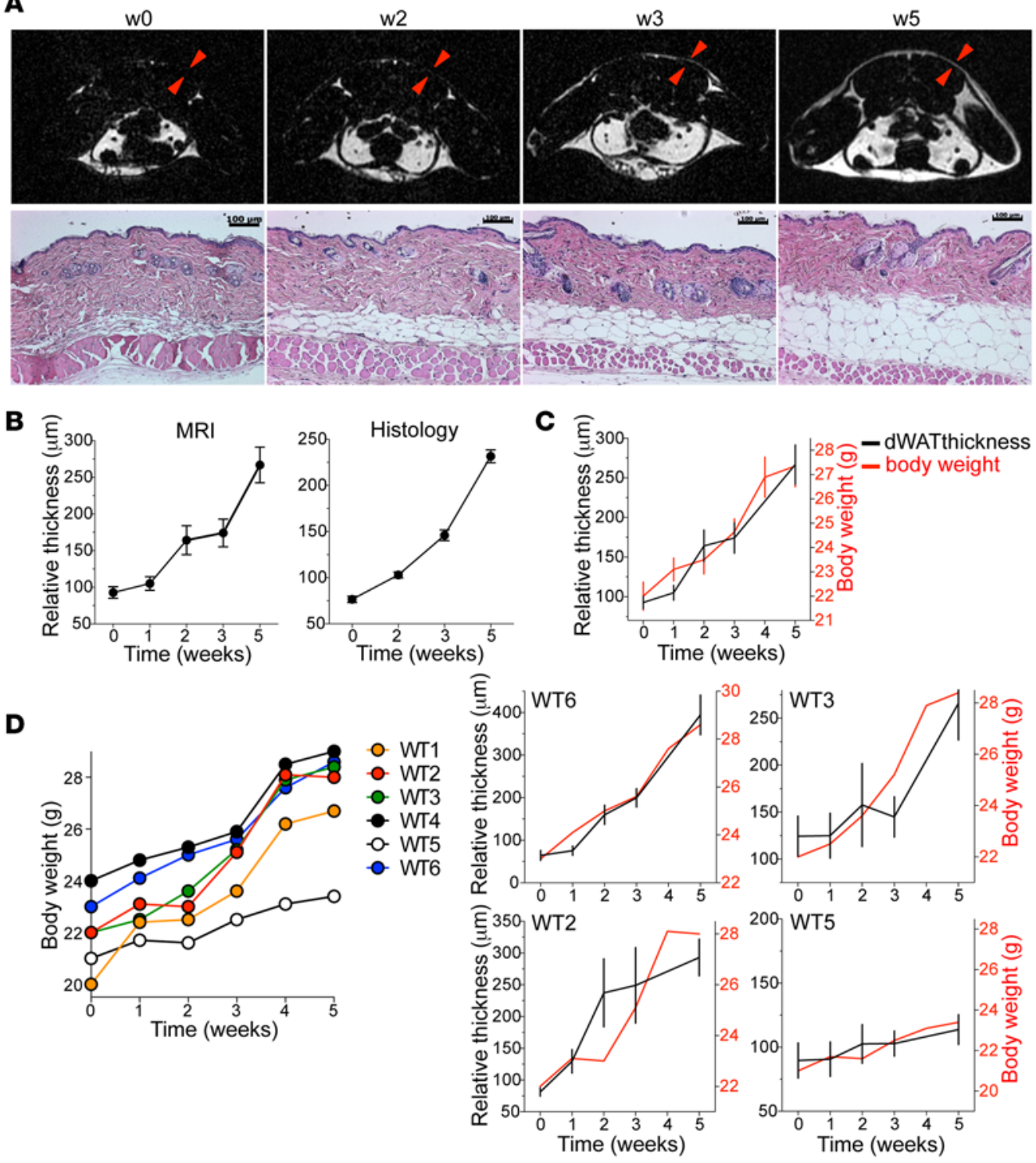

Figure 6. Longitudinal assay of accumulating dermal WAT in mice fed a high-fat diet. (A) MR images of approximately equivalent abdominal sections from the same mouse fed a high-fat diet (week 0 through week 5; w0, w2, w3, w5; dWAT is shown bracketed by red arrowheads). Samples from corresponding mice were collected and analyzed by histological analysis (below). Scale bars: $100 \mu \mathrm{m}$. (B) Thickness of dWAT was assayed by MRI during the course of high-fat feeding ( $n=$ 6) and compared with the thickness assessed by histological assay of corresponding mice (data is pooled from ventral and dorsal, anagen and nonanagen skins). (C) The kinetics of dWAT expansion is compared with body weight. (D) Variability in the rate of accumulation of body weight is illustrated for a cohort of 6 mice fed a high-fat diet (left side) and compared with the accrual of dWAT (right side, for the 4 mice indicated). Dot plots show mean values; error bars indicate \pm SEM. Statistical analysis was performed with unpaired 1-tailed $t$ tests using GraphPad Prism software.

neutral temperatures may determine the impact of dWAT on health and physiology. We hypothesize that subjects with thin insulation will be more susceptible to BAT activation, and vice versa. Perhaps related to this, recent studies of healthy male volunteers noted considerable variability between subjects with respect to their susceptibility to BAT activation $(23,24)$. Since BAT activation has been proposed by many studies to be prohealth and antiinflammatory $(6-8,25-27)$, assay of skin-associated lipid may be an independent variable that could be routinely measured (25).

Compared with mice, where dWAT is distinguished from sWAT by a muscle layer (the panniculus carnosus), human skin lacks this delimiting muscle layer for the majority of body sites (1-3). Human s.c. adipose tissues have been divided into deep and superficial layers - separated by fascia — and their func- 

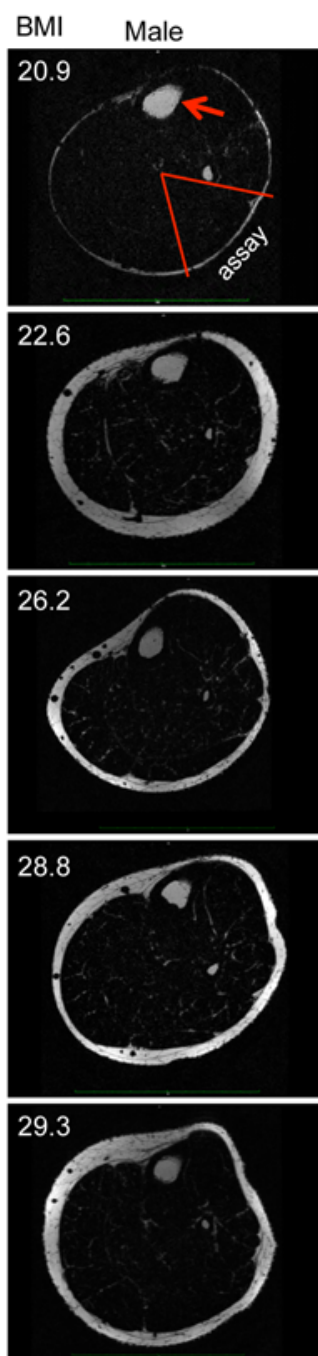
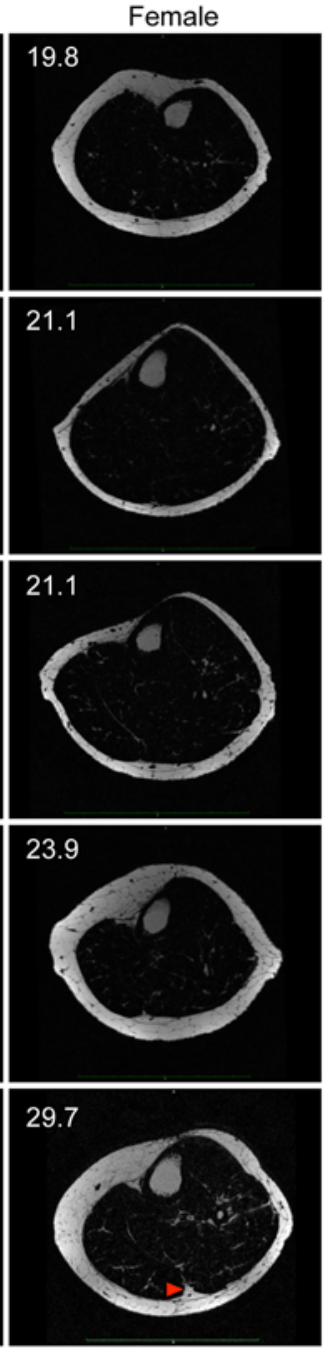
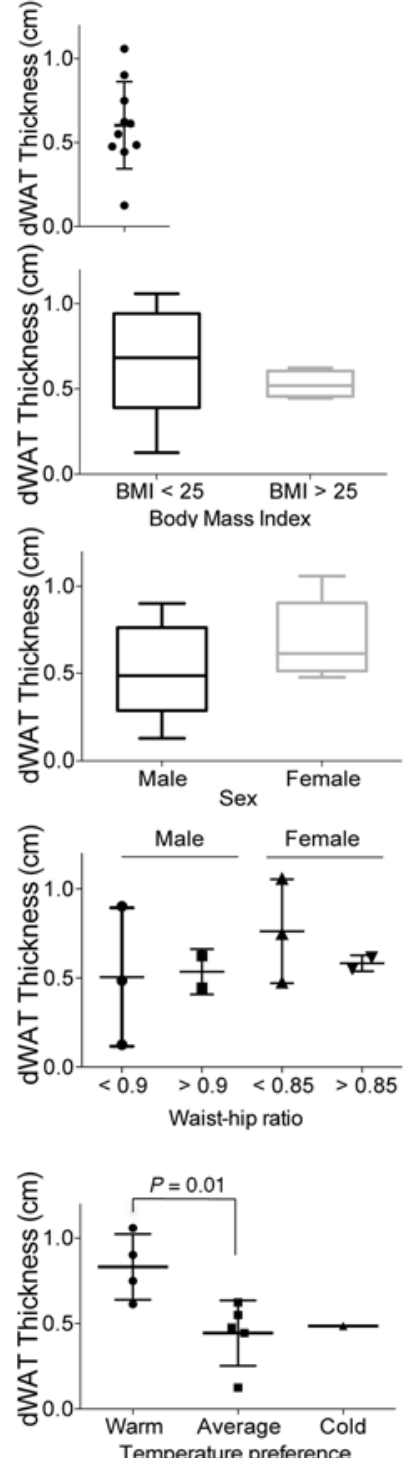

Figure 7. MRI evaluation of dWAT in a cohort of normal human subjects. An 8-channel extremity coil array was wrapped around the calf of 10 human subjects and used to assess the skin-associated lipid. The images derived from each subject are ordered top to bottom with respect to BMI (top left number; same images ordered with respect to waist/hip ratio are shown in Supplemental Figure 3). The strategy applied for consistent sampling is indicated (red arc) and uses the lipid-laden BM as a reference point (indicated with a red arrow). Intramuscular fat is indicated with a red arrowhead. The variability of dermal white adipose tissue (dWAT) thickness is shown in the top graph; other preliminary analysis of potential correlations are also shown, including BMI, sex, and waist/hip ratio for males and females. Subjects were asked to rate their temperature preference relative to their working/home cohorts, either warm, average, or cold. Dot plots show mean values; error bars indicate \pm SEM. Box-and-whisker plots were presented, and statistical analysis was performed with unpaired 1-tailed $t$ tests using GraphPad Prism software. Dot plots are presented as for Figure 3; box-and-whisker plots are presented as described in Figure 2.

tionality and regulation is known to be different $(22,28,29)$. The deep layers vary with BMI and body site, whereas the superficial layer is evenly distributed, suggesting that this superficial layer could be the human equivalent of dWAT. For most body sites (for example thoracic and abdominal sites), the lack of demarcation may present a difficulty of interpretation; it is likely that the properties of dWAT and sWAT will be sufficiently different to merit separate measurement. For example, thermogenically active fat depots such as sWAT show extensive lipolysis and vasodilation upon $\beta 3$-adrenergic stimulation $(30,31)$. Both of these properties are likely to reduce the insulating properties of a fat depot, and therefore, both are unlikely to be features of dWAT. Indeed, bringing warm blood to the body surface in obese subjects may generate the sen- 
sation of cold. Assessment of calf skin-associated fat has been used before as a measure of s.c. fat; fat was redistributed from an s.c. layer to an intramuscular location in patients with familial partial lipodystrophy (32). Future studies will define a dWAT-specific marker (or reaction) to conclusively identify this insulator dWAT depot in human subjects.

The technique we describe uses the different resonant frequencies of water and fat (chemical shift effect in MRI) to estimate the signals from water and fat, which are then separated into fat-only and water-only images. Lunati et al. (33) illustrated the contrasting fat and water images for MRI images of transverse slices of rat subjects; this early study also showed a separate layer of skin-associated fat. Fat and water images can be combined to generate fat-fraction images, which are often used clinically to provide an image with high dynamic range for a variety of tissue types or to calculate the absolute concentration of fat within a voxel. This method is also internally calibrated for comparison of data across platforms and sites. Other studies based on proton-density fat fraction (PDFF) images (using gradient echo acquisitions) provide absolute readings able to discriminate BAT and WAT $(27,34,35)$.

Instead of applying fat fraction, we used fat-only signals, since this modality allowed higher resolution and visualization of the thin layer of dWAT, particularly in rodents, with the simultaneous measurement of BAT and WAT. This method therefore compromises on the assay of absolute lipid density and provides instead the relative concentration of lipid. To achieve ultrahigh-resolution images of fat in skin, Querleux and colleagues developed a dedicated gradient surface coil, which they added onto the typical MRI instrumentation. This methodology was able to reveal the structure and number of hair follicles, cellulitic seams, sebaceous glands, and the substructure of lesions $(36,37)$. Other investigators have generated high-resolution methods for determining the absolute fat content of BAT, along with total volume, for both human subjects and rodents $(27,34,35,38-40)$. Although volume and lipid density provide a snapshot of total BAT and the level of intra-adipocyte lipid reserves, they do not provide a quantitative estimate of susceptibility to thermogenesis or total thermogenic capacity; these functional markers are under development (25).

In conclusion, this work has demonstrated the ability of CSE MRI to enable comprehensive assessment of BAT and WAT, together with the newly defined dWAT layer. There is considerable interest in defining the role of BAT activation in the development of obesity, since BAT consumes calories. More recently, the positive effects of BAT on health outcomes - whether diabetes, inflammatory obesity, or cancer - have been suggested to reflect the endocrine activities of activated BAT $(41,42)$ or auxiliary activities such as regulation of insulin sensitivity (43). Our previous study of a genetic model of underinsulation (4) suggests that the thickness of dWAT determines the relative activation of BAT, with all the associated implications. Whether for human subjects or for rodent models, this method will be an important tool for the holistic studies of diseases and molecules that affect the development of the dWAT skin-associated insulator layer and the thermogenic response in mammals.

\section{Methods}

Mice. All animals were housed at constant temperature $\left(20^{\circ} \mathrm{C}-23^{\circ} \mathrm{C}\right)$ in 12-hour light/dark cycles with free access to water and standard chow ad libitum (Harlan Teklad Global Diet, catalog 2018, for BALB/cJ mice and FormuLab diet, catalog 5008, from LabDiet for C57BL/6J mice). To induce obesity in C57BL/6J mice, their diet was switched to Harlan Teklad custom high-fat diet ( $45 \%$ calories from fat), catalog TD06415. BALB/cJ $S d c 1^{-/-}$mice have been described previously $(4,44)$; at the time of study, the control $\mathrm{BALB} / \mathrm{cJ}$ mice and $S d c 1^{-1-} \mathrm{BALB} / \mathrm{cJ}$ mice were age-matched and 8-10 weeks old. C57BL/6J ob/ob mice and C57BL/6J control mice were distributed by the Jackson Laboratory, bred in-house, and imaged when they were 7-11 weeks old. Data shown is from female mice, except for data panels that describe the results of high-fat diet administration, as high-fat diets were administered to male mice.

Imaging studies. For the MRI acquisitions, mice were either euthanized, stored at room temperature, and imaged within 1 hour, or they were anesthetized with isoflurane. Mice were placed in a prone position in an 8-channel wrist coil array (Invivo). All MRI experiments were carried out using a 3T system (MR750, DV24.0, GE Healthcare). Fat-water separated imaging was performed using a chemical shift encoded (CSE) fast spin echo (FSE) 2D multislice technique, including the following typical acquisition parameters: repetition time: $814 \mathrm{~ms}$; echo time: $23.8 \mathrm{~ms}$; 70 slices; slice thickness: $0.6-0.7 \mathrm{~mm}$; spacing (gap) between slices: $0.1 \mathrm{~mm}$; cranial-caudal orientation; field of view: $[80 \times 40]-[100 \times 50] \mathrm{mm}^{2}$; voxel dimensions: $[0.25 \times 0.31 \times 0.60]-[0.31 \times 0.39 \times 0.70] \mathrm{mm}^{3} ;$ receiver bandwidth: $\pm 62.5 \mathrm{kHz}$; number of averages: 1 ; 
total acquisition time: $7 \mathrm{~min}, 30 \mathrm{sec}$; and no parallel imaging acceleration. Three echo shifts $(-0.2 \mathrm{~ms}, 0.6$ $\mathrm{ms}$, and $1.4 \mathrm{~ms}$ ) were acquired to enable fat-water separation with optimal signal/noise ratio $(10,45)$. From these CSE acquisitions, water-only and fat-only images were reconstructed online using the iterative decomposition of water and fat with echo asymmetry and least-squares estimation (IDEAL) algorithm (10, 46) using 3D OsiriX software (v3.9.3 32-bit). The consistency of the signal across the coil diameter was assessed using corn oil phantom measurements (Supplemental Figure 1).

Image Analysis. For imaging of mice, iBAT depot, perigonadal WAT depot, and other WAT depots, including mammary glands, were delineated based on anatomical location. The average fat signal intensity was calculated across the entire depot over multiple slices, with 3-6 slices covering the entire depot.

For depots other than dermal WAT, multiple circular ROIs were manually drawn within the depots on the reconstructed MRI slices in each animal. In these depots, 5 ROIs were measured per slice and were uniformly distributed throughout the tissue of interest, with ROI size of $0.2 \mathrm{~mm}^{2}$.

Dorsal WAT and ventral dWAT were located based on anatomical location. Within each reconstructed slice, a total of 20 rectangular ROIs (typical size of $1.0 \mathrm{~mm}^{2}$ ) were drawn on the dWAT: 10 ROIs uniformly covering the dorsal dWAT, and 10 ROIs uniformly covering the ventral dWAT. In placing the ROIs, particular care was taken to avoid inclusion of any confounding signals from the sWAT that may overlay the iBAT depot or exist in close proximity of the dWAT layer. Using these ROI measurements, the average dWAT thickness in dorsal and ventral regions was estimated for each animal, using the following approximation:

$h_{\mathrm{dWAT}} \approx\left(\mathrm{s}_{\mathrm{dWAT}} \times h_{\mathrm{ROI}}\right) / \mathrm{s}_{\mathrm{WAT}}$

where $\mathrm{s}_{\mathrm{dWAT}}$ is the average signal intensity measured in the dWAT ROI, $h_{\mathrm{ROI}}$ is the height of the ROI covering the dWAT layer, and $\mathrm{s}_{\mathrm{WAT}}$ is the average signal intensity in a nearby visceral WAT depot (used as a reference, e.g., to compensate for spatially varying coil sensitivities, with the assumption that WAT is composed nearly entirely of adipose tissue). The sensitivity of this method was limited by the signal/noise ratio when voxels were partially filled, giving an estimated threshold of $58 \mu \mathrm{m}$ thickness for accurate quantitation of dWAT (further information can be found in Supplemental Methods).

Volume rendering analysis was performed using Mimics 18.00.525 software, applying a predefined threshold (739-3,045) for the segmentation mask.

Histological Analysis. Immediately following the imaging studies, skin, iBAT, perigonadal WAT, and mammary glands were dissected for histological processing. Samples were paraformaldehyde fixed (4\%) overnight and then paraffin embedded for evaluation. Tissue sections were deparaffinized, rehydrated, and stained with standard H\&E protocol for visualization.

Human Volunteer Study. In addition, 10 healthy volunteers were scanned in accordance with the local IRB after informed consent was obtained. The volunteers were scanned at $3 \mathrm{~T}$ in the same magnet as the mice, using an 8-channel phased array extremity coil (Invivo). Similar to the mouse experiments, fat-waterseparated imaging was performed using a CSE FSE 2D multislice technique, with the following parameters: repetition time: $793 \mathrm{~ms}$; echo time: $20.4 \mathrm{~ms}$; 60 slices; slice thickness: $0.8 \mathrm{~mm}$; spacing between slices: $0.2 \mathrm{~mm}$; axial orientation; field of view: $[140 \times 140]-[150 \times 150] \mathrm{mm}^{2}$; voxel dimensions: $[0.44 \times 0.55 \times$ $0.80]-[0.47 \times 0.59 \times 0.80] \mathrm{mm}^{3}$; receiver bandwidth: $\pm 39 \mathrm{kHz}$; number of averages: 1 ; total acquisition time: $10 \mathrm{~min}, 29 \mathrm{sec}$; no parallel imaging acceleration; and 3 echo shifts $(-0.2 \mathrm{~ms}, 0.6 \mathrm{~ms}$, and $1.4 \mathrm{~ms})$ to enable optimal fat-water separation $(10,45)$. The axial slices were centered around the widest diameter of the calf. Water-only and fat-only images were reconstructed as for the mouse acquisitions.

Measurement of skin-associated fat thickness in the human volunteer study was performed from the fat-only images. Note that, unlike the mouse scans, the acquired spatial resolution is sufficient for direct measurement of the thickness of this layer in humans. On each subject, thickness measurements were performed on a single slice near the center of the volume, which was chosen to avoid intermuscular fat adjacent to the skin-associated fat layer. Fifteen thickness measurements were made on this slice, covering a left-posterior sector of the calf (see Figure 5). The final fat thickness measurement for each volunteer was obtained by averaging these 15 individual measurements.

Statistics. Data are expressed as mean \pm SEM, and statistical analysis was performed with unpaired 1 -tailed $t$ tests using GraphPrism6 software. $P$ values less than 0.05 were considered statistically significant.

Study approval. All experiments that included the use of mice were approved by the University of Wisconsin IACUC (Institutional assurance number for University of Wisconsin-Madison A-3368-01), and studies were performed in strict accordance with the recommendations in the Guide for the Care and Use of 
Laboratory Animals (National Academies Press. 2011.). All imaging studies that include the use of human volunteers were HIPAA compliant and were performed after obtaining approval from the Health Sciences IRB at the University of Wisconsin-Madison. A total of 10 healthy volunteers ( 5 men and 5 women, age range $22-41$, mean 32.9 years) were prospectively recruited after obtaining informed written consent.

\section{Author contributions}

CMA developed the strategy for this study; identified resources; consulted on technical aspects, data analysis, and presentation; and prepared the manuscript. IK designed and executed the experiments, prepared and presented the data, and consulted on manuscript design and presentation. DH designed the imaging techniques and consulted on data analysis and presentation and manuscript preparation. ARA performed the 3D data analysis. SBR consulted on experimental design, the imaging protocol, and manuscript development.

\section{Acknowledgments}

We thank Brian W. Parks (Department of Nutritional Sciences) for his advice and assistance with the study of diet-induced obesity, and also Margaret Wheathers for her assistance with the analysis of 3D renderings of adipose depots. Thanks also for the gracious permission from S. Cinti (and journal editors at Proceedings of the Nutrition Society for reproduction of the dissection shown in Figure 3B. The authors wish to acknowledge support from the NIH (UL1TR00427, R01 DK083380, R01 DK088925, R01 DK100651, and K24 DK102595), as well as GE Healthcare, which provides research support to the University of Wisconsin.

Address correspondence to: Caroline M. Alexander, McArdle Laboratory, School of Medicine and Public Health, WIMR2, 1111 Highland Avenue, University of Wisconsin-Madison. Phone: 608.265.5182. E-mail: alexander@oncology.wisc.edu.

1. Alexander CM, et al. Dermal white adipose tissue: a new component of the thermogenic response. J Lipid Res. 2015;56(11):2061-2069.

2. Driskell RR, Jahoda CA, Chuong CM, Watt FM, Horsley V. Defining dermal adipose tissue. Exp Dermatol. 2014;23(9):629-631.

3. Schneider MR. Coming home at last: dermal white adipose tissue. Exp Dermatol. 2014;23(9):634-635.

4. Kasza I, et al. Syndecan-1 is required to maintain intradermal fat and prevent cold stress. PLoS Genet. 2014;10(8):e1004514.

5. Bachman ES, et al. betaAR signaling required for diet-induced thermogenesis and obesity resistance. Science. 2002;297(5582):843-845.

6. Cannon B, Nedergaard J. Brown adipose tissue: function and physiological significance. Physiol Rev. 2004;84(1):277-359.

7. Harms M, Seale P. Brown and beige fat: development, function and therapeutic potential. Nat Med. 2013;19(10):1252-1263

8. Bartelt A, Heeren J. Adipose tissue browning and metabolic health. Nat Rev Endocrinol. 2014;10(1):24-36.

9. Kozak LP. Brown fat and the myth of diet-induced thermogenesis. Cell Metab. 2010;11(4):263-267.

10. Reeder SB, et al. Iterative decomposition of water and fat with echo asymmetry and least-squares estimation (IDEAL): application with fast spin-echo imaging. Magn Reson Med. 2005;54(3):636-644.

11. Cinti S. The adipose organ: morphological perspectives of adipose tissues. Proc Nutr Soc. 2001;60(3):319-328.

12. Sidossis L, Kajimura S. Brown and beige fat in humans: thermogenic adipocytes that control energy and glucose homeostasis. $J$ Clin Invest. 2015;125(2):478-486.

13. Wu J, et al. Beige adipocytes are a distinct type of thermogenic fat cell in mouse and human. Cell. 2012;150(2):366-376.

14. Fellmann L, Nascimento AR, Tibiriça E, Bousquet P. Murine models for pharmacological studies of the metabolic syndrome. Pharmacol Ther. 2013;137(3):331-340.

15. Fischer AW, et al. Leptin Raises Defended Body Temperature without Activating Thermogenesis. Cell Rep. 2016;14(7):16211631.

16. Lee MJ, Wu Y, Fried SK. Adipose tissue heterogeneity: implication of depot differences in adipose tissue for obesity complications. Mol Aspects Med. 2013;34(1):1-11.

17. Fu J, Hofker M, Wijmenga C. Apple or pear: size and shape matter. Cell Metab. 2015;21(4):507-508.

18. Koza RA, et al. Changes in gene expression foreshadow diet-induced obesity in genetically identical mice. PLoS Genet. 2006;2(5):e81

19. Dalgaard K, et al. Trim28 Haploinsufficiency Triggers Bi-stable Epigenetic Obesity. Cell. 2016;164(3):353-364.

20. Montgomery MK, et al. Mouse strain-dependent variation in obesity and glucose homeostasis in response to high-fat feeding. Diabetologia. 2013;56(5):1129-1139.

21. Parks BW, et al. Genetic architecture of insulin resistance in the mouse. Cell Metab. 2015;21(2):334-346.

22. Shen W, et al. Adipose tissue quantification by imaging methods: a proposed classification. Obes Res. 2003;11(1):5-16.

23. van Marken Lichtenbelt WD, et al. Cold-activated brown adipose tissue in healthy men. N Engl J Med. 2009;360(15):1500-1508.

24. Chondronikola M, et al. Brown adipose tissue improves whole-body glucose homeostasis and insulin sensitivity in humans. Diabetes. 2014;63(12):4089-4099.

25. Cypess AM, Haft CR, Laughlin MR, Hu HH. Brown fat in humans: consensus points and experimental guidelines. Cell Metab. 2014;20(3):408-415.

26. Seale P, Kajimura S, Spiegelman BM. Transcriptional control of brown adipocyte development and physiological function--of 
mice and men. Genes Dev. 2009;23(7):788-797.

27. Bauwens M, et al. Molecular imaging of brown adipose tissue in health and disease. Eur J Nucl Med Mol Imaging. 2014;41(4):776-791

28. Kelley DE, Thaete FL, Troost F, Huwe T, Goodpaster BH. Subdivisions of subcutaneous abdominal adipose tissue and insulin resistance. Am J Physiol Endocrinol Metab. 2000;278(5):E941-E948.

29. Walker GE, et al. Deep subcutaneous adipose tissue: a distinct abdominal adipose depot. Obesity (Silver Spring). 2007;15(8):1933-1943.

30. Lim S, et al. Cold-induced activation of brown adipose tissue and adipose angiogenesis in mice. Nat Protoc. 2012;7(3):606-615

31. Seale $\mathrm{P}$, et al. Prdm16 determines the thermogenic program of subcutaneous white adipose tissue in mice. J Clin Invest. 2011;121(1):96-105

32. Addison O, Marcus RL, Lastayo PC, Ryan AS. Intermuscular fat: a review of the consequences and causes. Int J Endocrinol. 2014;2014:309570.

33. Lunati E, Marzola P, Nicolato E, Fedrigo M, Villa M, Sbarbati A. In vivo quantitative lipidic map of brown adipose tissue by chemical shift imaging at 4.7 Tesla. J Lipid Res. 1999;40(8):1395-1400.

34. Smith DL, Yang Y, Hu HH, Zhai G, Nagy TR. Measurement of interscapular brown adipose tissue of mice in differentially housed temperatures by chemical-shift-encoded water-fat MRI. J Magn Reson Imaging. 2013;38(6):1425-1433.

35. Hu HH, Smith DL, Nayak KS, Goran MI, Nagy TR. Identification of brown adipose tissue in mice with fat-water IDEAL-MRI. J Magn Reson Imaging. 2010;31(5):1195-1202.

36. Querleux B. Magnetic resonance imaging and spectroscopy of skin and subcutis. J Cosmet Dermatol. 2004;3(3):156-161.

37. Querleux B, Cornillon C, Jolivet O, Bittoun J. Anatomy and physiology of subcutaneous adipose tissue by in vivo magnetic resonance imaging and spectroscopy: relationships with sex and presence of cellulite. Skin Res Technol. 2002;8(2):118-124.

38. Hamilton G, Smith DL, Bydder M, Nayak KS, Hu HH. MR properties of brown and white adipose tissues. J Magn Reson Imaging. 2011;34(2):468-473

39. Chen YC, et al. Measurement of human brown adipose tissue volume and activity using anatomic MR imaging and functional MR imaging. J Nucl Med. 2013;54(9):1584-1587.

40. Branca RT, et al. Detection of brown adipose tissue and thermogenic activity in mice by hyperpolarized xenon MRI. Proc Natl Acad Sci U S A. 2014;111(50):18001-18006.

41. Villarroya J, Cereijo R, Villarroya F. An endocrine role for brown adipose tissue?. Am J Physiol Endocrinol Metab. 2013;305(5):E567-E572.

42. Wang GX, Zhao XY, Lin JD. The brown fat secretome: metabolic functions beyond thermogenesis. Trends Endocrinol Metab. 2015;26(5):231-237.

43. Stanford KI, et al. Brown adipose tissue regulates glucose homeostasis and insulin sensitivity. J Clin Invest. 2013;123(1):215-223

44. McDermott SP, Ranheim EA, Leatherberry VS, Khwaja SS, Klos KS, Alexander CM. Juvenile syndecan-1 null mice are protected from carcinogen-induced tumor development. Oncogene. 2007;26(10):1407-1416.

45. Pineda AR, Reeder SB, Wen Z, Pelc NJ. Cramér-Rao bounds for three-point decomposition of water and fat. Magn Reson Med. 2005;54(3):625-635

46. Reeder SB, et al. Multicoil Dixon chemical species separation with an iterative least-squares estimation method. Magn Reson Med. 2004;51(1):35-45. 